\title{
Apresentação Clínica de Pacientes Obesos com Diagnóstico Polissonográfico de Apnéia Obstrutiva do Sono
}

\section{artigo original}

Paloma B. Gregório

RODRIGO A. ATHANAZIO

ALMIR G.V. BITENCOURT

FLÁVIA B.C. SERRA NEVES

Carla Daltro

ERIVALDO ALVES

FRANCISCO HORA

Instituto Cardiopulmonar e

Clínica Otorrinocenter do Laboratório do Sono, e Grupo de Obesidade e Cirurgia do Hospital Português da Bahia,

Salvador, BA.

Recebido em 29/01/07 Revisado em 02/04/07 Aceito em 23/04/07

\section{RESUMO}

Objetivo: Avaliar a sintomatologia de pacientes obesos com diagnóstico polissonográfico de Síndrome da Apnéia/Hipopnéia Obstrutiva do Sono (SAHOS). Métodos: Foram avaliados todos os pacientes obesos (IMC $\geq 30$ $\mathrm{kg} / \mathrm{m}^{2}$ ) que realizaram polissonografia em dois laboratórios de sono da cidade de Salvador, BA, no período de julho a dezembro de 2004 e apresentaram Índice de Apnéia/Hipopnéia (IAH) $\geq 5 \mathrm{ev} / \mathrm{h} / \mathrm{sono}$. Os sintomas considerados foram sonolência excessiva diurna, despertares noturnos, sensação de engasgos e/ou sufocamentos durante o sono. Resultados: Foram incluídos 73 pacientes, sendo $57,5 \%$ do sexo masculino com média de idade e IMC de, respectivamente, 45,2 anos e $38,0 \mathrm{~kg} / \mathrm{m}^{2}$. A maioria $(49,3 \%)$ foi classificada como SAHOS grave (IAH $\geq 30$ ). Os dados revelaram que $19,2 \%$ destes pacientes não apresentavam nenhum dos sintomas pesquisados, $28,8 \%$, apenas um sintoma, $30,1 \%$, dois e $21,9 \%$, três. Não houve diferença estatisticamente significante em relação à gravidade da doença entre os indivíduos com e sem sintomas. Houve uma maior freqüência de pacientes assintomáticos entre os pacientes com obesidade severa $(30,8 \%$ versus $5,9 \% ; p=0,007)$. Conclusões: A polissonografia parece ser um exame fundamental na avaliação de pacientes obesos devido à alta taxa de indivíduos assintomáticos com índices polissonográficos alterados neste grupo, em especial nos obesos graves. (Arq Bras Endocrinol Metab 2007;51/7:1064-1068)

Descritores: Apnéia do sono; Obesidade; Polissonografia; Cirurgia bariátrica

\section{ABSTRACT}

Clinical Presentation of Obese Patients with Polysomnographic Diagnosis of Obstructive Sleep Apnea.

Aim: To evaluate the symptoms of obese patients with polysomnographic diagnosis of obstructive sleep apnea (OSA). Methods: All obese patients $\left(\mathrm{BMI} \geq 30 \mathrm{~kg} / \mathrm{m}^{2}\right)$ that accomplished overnight polysomnography in two sleep laboratories in the city of Salvador, BA, Brazil, in the period of July to December of 2004, and had an apnea hypopnea index (AHI) $\geq 5$. The considered symptoms were excessive daytime sleepiness, awakenings during the night and nocturnal choking or gasping. Results: 73 patients were included, being $57.5 \%$ male, with mean age and BMI of 45.2 years old and $38.0 \mathrm{~kg} / \mathrm{m}^{2}$, respectively. The majority $(49.3 \%)$ was classified as having severe OSA (AHI $\geq 30$ ). Data revealed that $19.2 \%$ of the patients did not have any symptoms, while $28.8 \%$ had one symptom, $30.1 \%$ had two and $21.9 \%$ had three. There was no statistically significant difference in the severity of OSA between the subjects with and without symptoms. There was a higher prevalence of asymptomatic patients in the subgroup with severe obesity - BMI $\geq 35 \mathrm{~kg} / \mathrm{m}^{2}(30.8 \%$ vs. $5.9 \% ; p=0.007)$. Conclusions: The polysomnography seems to be a fundamental exam in the evaluation of obese patients due to the elevated prevalence of asymptomatic individuals with OSA in this group, especially the severe obese. (Arq Bras Endocrinol Metab 2007;51/7:1064-1068)

Keywords: Sleep apnea; Obesity; Polysomnography; Bariatric surgery 
$\mathrm{A}^{\mathrm{n}}$ PREVAlÊnCIA de Síndrome da Apnéia/ Hipopnéia Obstrutiva do Sono (SAHOS) tem aumentado bastante nas últimas décadas, em paralelo à recente epidemia mundial de obesidade (1). Estima-se que, em adultos de países ocidentais, $4 \%$ dos homens e $2 \%$ das mulheres apresentem SAHOS clinicamente significante $(2,3)$. Mesmo havendo um maior reconhecimento deste problema pelos médicos, em especial após o advento da polissonografia, a maioria dos indivíduos afetados permanecem sem diagnóstico.

A fisiopatologia da SAHOS está intimamente relacionada à obesidade, de forma que $60 \%$ a $90 \%$ dos indivíduos com SAHOS têm índice de massa corpórea (IMC) $>29 \mathrm{~kg} / \mathrm{m}^{2}$, além de haver relação direta entre a SAHOS e as classes de obesidade (4-6). Considerações anatômicas e funcionais das vias aéreas superiores, do sistema nervoso central e dos níveis séricos de leptina interagem para o desenvolvimento da SAHOS em obesos (7).

O diagnóstico desse distúrbio do sono requer atenção, visto seu impacto em um espectro de distúrbios neurocognitivos agudos e crônicos e alterações cardiovasculares, incluindo arritmias, hipertensão arterial sistêmica e pulmonar (8). Estudo recente demonstrou, ainda, que a SAHOS aumenta significativamente o risco de acidente vascular cerebral e mortalidade por qualquer causa, independentemente de outros fatores de risco, como hipertensão (9). Além disso, adultos obesos com SAHOS submetidos a procedimentos cirúrgicos maiores podem apresentar diversas complicações no manejo das vias aéreas no período peri e pósoperatório (8).

Cada vez mais a cirurgia bariátrica tem sido utilizada para o tratamento da obesidade mórbida. Entretanto, ainda não está bem definida a importância da polissonografia (PSG) como exame pré-operatório de rotina nesses pacientes (10). O objetivo deste estudo é avaliar a sintomatologia de pacientes obesos com diagnóstico polissonográfico de SAHOS, a fim de determinar se existe a necessidade obrigatória deste exame nos pacientes que se submeterão à cirurgia bariátrica independentemente da presença de sintomas compatíveis com a SAHOS.

\section{MATERIAL E MÉTODOS}

Foram avaliados todos os pacientes obesos (IMC $\geq 30$ $\mathrm{kg} / \mathrm{m}^{2}$ ) que realizaram polissonografia em dois laboratórios de sono da cidade de Salvador, BA, no período de julho a dezembro de 2004 e que obtiveram índice de apnéia/ hipopnéia $(\mathrm{IAH}) \geq 5 \mathrm{ev} / \mathrm{h} /$ sono. Todos os pacientes foram submetidos a um questionário sobre dados clínicos que incluíam características sócio-demográficas, presença de sinais e sintomas relacionados a apnéia do sono; escala de sonolência diurna de Epworth; mensuração do peso e altura em balança mecânica (Filizola ${ }^{\circledR}$ modelo 131), com o paciente situado no centro da plataforma vestido e sem sapatos. O IMC foi calculado através da fórmula: peso em quilogramas dividido pela altura em metros, ao quadrado, tendo sido assim classificado (11): normal (18,5 a 24,9 $\left.\mathrm{kg} / \mathrm{m}^{2}\right)$; sobrepeso $\left(25,0\right.$ a $\left.29,9 \mathrm{~kg} / \mathrm{m}^{2}\right)$; obeso I (30,0 a $\left.34,9 \mathrm{~kg} / \mathrm{m}^{2}\right)$; obeso II $\left(35,0\right.$ a $\left.39,9 \mathrm{~kg} / \mathrm{m}^{2}\right)$ e obeso III $(\geq$ $\left.40,0 \mathrm{~kg} / \mathrm{m}^{2}\right)$.

Considerou-se assintomático aquele indivíduo que negava sonolência diurna, sufocamento noturno e despertares noturnos. O ronco, por ser um sintoma com alta sensibilidade e baixa especificidade, não foi considerado positivo quando apresentado de forma isolada. A Escala de Sonolência de Epworth foi utilizada para avaliar a presença de sonolência diurna, sendo a mesma considerada alterada quando o escore fosse $\geq 10$ (12).

As polissonografias foram realizadas através do equipamento computadorizado Sonolab 620, sendo o laudo revisto por um único especialista. $\mathrm{O}$ exame foi conduzido durante toda a noite, em sono espontâneo, sem nenhuma sedação ou privação do sono. Foram registrados: eletroencefalograma (eletrodos $\mathrm{C} 3, \mathrm{C} 4)$, oculograma $(\mathrm{O} 1, \mathrm{O} 2)$, eletromiograma (eletrodos nas regiões mentoniana, submentoniana e MMII), eletrocardiograma, fluxo aéreo (cânula e termister nasal), esforço respiratório (cinta torácica e abdominal), ronco (microfone no queixo) e posição do corpo (sensor de posição). A saturação da oxi-hemoglobina foi medida através da oximetria de pulso (13). O estagiamento do sono seguiu os critérios adotados de Rechtschaffen e Kalles (14). Os eventos respiratórios foram assim definidos: redução de $50 \%$ ou mais da amplitude de uma medida da ventilação pulmonar validada durante o sono a partir de um valor basal (hipopnéia) ou a sua completa cessação (apnéia), ou evidente redução da amplitude ventilatória, obtida através de uma medida validada, que ocorre durante o sono, não alcançando os critérios acima, porém com um decréscimo superior ou igual a $3 \%$ na saturação da oxi-hemoglobina e/ou a um microdespertar; todos estes eventos com duração igual ou superior a 10 segundos (15).

O IAH foi obtido através de exame polissonográfico, dividindo o total de eventos respiratórios pelas horas de sono (15). Os pacientes foram classificados de acordo com o IAH em: não apnéicos - menos de 5,0 eventos/hora de sono; com apnéia leve - entre 5,0 e 15 eventos/hora de sono; com apnéia moderada - entre 15,1 e 30,0 eventos por/hora de sono e com apnéia grave - mais de 30,0 eventos/hora de sono (15).

O presente estudo foi aprovado pelo Comitê de Ética em Pesquisa em Seres Humanos do Hospital Português. A análise estatística foi realizada através do programa SPSS 9.0. As variáveis contínuas foram apresentadas sob a forma de média \pm desvio-padrão acrescidos da mediana e amplitude interquartil quando não possuíam distribuição normal, e as categóricas, sob a forma de freqüência relativa. Utilizou-se 
teste qui-quadrado para comparação de variáveis categóricas. A comparação entre médias foi realizada através do teste $t$ de Student, para as variáveis contínuas com distribuição normal, e do teste de Mann-Whitney, para as variáveis contínuas sem distribuição normal. Todos os testes foram bicaudais e considerou-se estatisticamente significante quando $\mathrm{p}<0,05$.

\section{RESULTADOS}

Foram estudados 73 pacientes com média de idade de $45,2 \pm 12,4$ anos (variando de 21 a 76 anos), sendo $57,5 \%$ do sexo masculino. O IMC variou de 30,1 a $63,7 \mathrm{~kg} / \mathrm{m}^{2}$, com média de $38,0 \pm 7,5 \mathrm{~kg} / \mathrm{m}^{2}$, sendo que 39 indivíduos $(53,4 \%)$ apresentavam obesidade grau II ou III (IMC $\left.\geq 35 \mathrm{~kg} / \mathrm{m}^{2}\right)$. A média do IAH e da Escala de Epworth foram de, respectivamente, 39,0 $\pm 29,5(6,9-115,9)$ e $10,2 \pm 5,1(0-22)$. Presença de distúrbio leve do sono foi encontrada em $21,9 \%$ dos pacientes, moderado em $28,8 \%$ e grave em $49,3 \%$.

Quanto aos sintomas avaliados, os dados revelaram que $14(19,2 \%)$ pacientes não apresentavam nenhum dos sintomas pesquisados (assintomáticos), 21 $(28,8 \%)$ apenas um sintoma, $22(30,1 \%)$ dois e 16 $(21,9 \%)$ os três. O sintoma mais comum foi sonolência diurna $(60,0 \%)$, seguido de despertar noturno $(56,9 \%)$ e sufocamento noturno $(41,7 \%)$. Houve um aumento na média do IAH nos pacientes com 2 ou 3 sintomas, em relação aos indivíduos assintomáticos ou com apenas um sintoma (tabela 1 ), no entanto essa diferença não foi estatisticamente significante $(\mathrm{p}=0,154)$.

A tabela 2 mostra a comparação dos dados entre os pacientes de acordo com o IMC. Os pacientes com obesidade grau I eram mais velhos e com maior prevalência de homens. Não houve diferença nos dois grupos em relação ao $\mathrm{IAH}$, à graduação do distúrbio do sono e ao valor da Escala de Epworth. Houve uma maior frequiência de pacientes assintomáticos entre os pacientes com obesidade grau II ou III $(30,8 \%$ versus $5,9 \% ; p=0,007)$. A tabela 3 demonstra a comparação dos dados entre os grupos de pacientes com e sem sintomas.

\section{DISCUSSÃO}

Nossos dados ressaltam que cerca de $20 \%$ dos pacientes obesos com IAH $\geq 5 \mathrm{ev} / \mathrm{h}$ não apresentam sintomatologia clássica relacionada à SAHOS. Entre esses obesos, aqueles com obesidade graus II ou III, que preenchem critérios (baseado no IMC e co-morbidades) para a indicação da cirurgia bariátrica, apresentaram um número ainda maior de indivíduos assintomáticos $(30 \%)$. Esses dados contribuem sobremaneira para a decisão de se realizar ou não a PSG como instrumento préoperatório de rotina em obesos que serão submetidos à cirurgia bariátrica. Na prática clínica, muitos médicos solicitam o exame apenas se o paciente apresentar sintomas clássicos relacionados à SAHOS. Entretanto, muitos pacientes obesos com distúrbio respiratório do sono são oligo ou assintomáticos.

A SAHOS é uma co-morbidade comum em pacientes obesos submetidos à cirurgia bariátrica e o seu manejo no peri e pós-operatório é complexo (10). Esses pacientes são, em geral, mais difíceis de serem intubados e têm maior risco de obstrução das vias aéreas após extubação (7). Os agentes anestésicos e analgésicos utilizados podem aumentar a severidade dos sintomas de SAHOS por diminuir a atividade dos músculos dilatadores da faringe, enquanto os opióides podem deprimir o centro respiratório e piorar a saturação de oxigênio (16). A hipotonia muscular que ocorre no período do sono REM aumenta o risco de complicações respiratórias durante o período pós-operatório inicial (17). A terapia com a pressão positiva contínua nas vias aéreas (CPAP - continuous positive airway pressure) otimizada minimiza o risco de complicações respiratórias e cardiovasculares relacionadas à SAHOS nesses pacientes (16). Este tratamento deve ser iniciado antes da cirurgia para minimizar complicações hemodinâmicas da SAHOS e adaptar o paciente com a ventilação não-invasiva. No entanto, o nível de CPAP necessário é muito variável e deve ser individualizado, tornando-se fundamental a confirmação e graduação desse diagnóstico no período pré-operatório a fim de iniciar uma terapêutica adequada (17).

Tabela 1. Valor do Índice de Apnéia/Hipopnéia (IAH) dos pacientes estudados de acordo com a sintomatologia.

\begin{tabular}{|c|c|c|}
\hline \multirow{2}{*}{$\begin{array}{l}\text { Presença dos Sintomas } \\
\text { (n) }\end{array}$} & \multicolumn{2}{|c|}{ Índice de Apnéia/Hipopnéia (IAH) } \\
\hline & Média \pm desvio-padrão & Mediana (AIO) \\
\hline Assintomático $(n=14)$ & $31,6 \pm 30,5$ & $17,5(15,2-40,2)$ \\
\hline 1 Sintoma $(n=21)$ & $35,4 \pm 31,5$ & $25,7(12,1-43,3)$ \\
\hline 2 Sintomas $(n=22)$ & $43,8 \pm 31,4$ & $34,5(18,1-68,3)$ \\
\hline 3 Sintomas $(n=16)$ & $43,6 \pm 23,2$ & $42,2(23,2-64,4)$ \\
\hline
\end{tabular}


Tabela 2. Características clínicas e demográficas dos pacientes estudados de acordo com o índice de massa corpórea (IMC).

\begin{tabular}{|c|c|c|c|}
\hline Características & IMC < $35(n=34)$ & $I M C \geq 35(n=39)$ & Valor $\mathbf{P}$ \\
\hline Idade (anos)* & $50,8 \pm 12,3$ & $40,3 \pm 10,3$ & $<0,001$ \\
\hline Gênero masculino (\%) & 73,5 & 43,6 & 0,017 \\
\hline $\mathrm{IAH}^{*}$ & $34,3 \pm 20,4$ & $43,0 \pm 35,4$ & 0,197 \\
\hline Mediana (AIQ) & $29,2(15,5-45,9)$ & $24,1(16,4-77,1)$ & \\
\hline Escala de Epworth* & $10,4 \pm 5,4$ & $10,0 \pm 4,8$ & 0,732 \\
\hline Distúrbio do sono & & & 0,653 \\
\hline Leve & $23,5 \%$ & $20,5 \%$ & \\
\hline Moderado & $23,5 \%$ & $33,3 \%$ & \\
\hline Grave & $52,9 \%$ & $46,2 \%$ & \\
\hline Freqüência de Sintomas & & & 0,007 \\
\hline Assintomático & $5,9 \%$ & $30,8 \%$ & \\
\hline 1 sintoma & $23,5 \%$ & $33,3 \%$ & \\
\hline 2 sintomas & $35,3 \%$ & $25,6 \%$ & \\
\hline 3 sintomas & $35,3 \%$ & $10,3 \%$ & \\
\hline
\end{tabular}

* Valores expressos em média \pm desvio-padrão.

Tabela 3. Características clínicas e demográficas dos pacientes de acordo com a sintomatologia.

\begin{tabular}{lccc}
\hline Características & $\begin{array}{c}\text { Assintomáticos } \\
\text { (n = 14) }\end{array}$ & $\begin{array}{c}\text { Sintomáticos } \\
\text { (n = 59) }\end{array}$ & Valor P \\
\hline Idade (anos)* & $39,4 \pm 9,8$ & $46,6 \pm 12,6$ & 0,049 \\
\hline Gênero Masculino (\%) & $35,7 \%$ & $62,7 \%$ & 0,066 \\
\hline IMC (kg/m²) & $41,1 \pm 6,1$ & $37,2 \pm 7,6$ & 0,021 \\
\hline Mediana (AIQ) & $40,6(38,2-43,5)$ & $34,60(31,2-41,0)$ & \\
IAH* & $31,6 \pm 30,5$ & $40,7 \pm 29,2$ & 0,163 \\
\hline Mediana (AIQ) & $17,5(15,2-40,2)$ & $31,8(16,4-63,3)$ & \\
Escala de Epworth* & $8,3 \pm 4,1$ & $10,6 \pm 5,2$ & 0,135 \\
\hline Distúrbio do sono & & & 0,121 \\
\hline Leve & $21,4 \%$ & $22,0 \%$ & \\
Moderado & $50,0 \%$ & $23,7 \%$ & \\
Grave & $28,6 \%$ & $54,2 \%$ & \\
\hline
\end{tabular}

* Valores expressos em média \pm desvio-padrão.

A PSG ainda é o "padrão ouro" para a avaliação dos distúrbios respiratório do sono. Trata-se de um exame complexo, caro, demorado, geralmente inconveniente para o paciente e limitado a alguns centros. Por este motivo, tem-se tentado criar uma maneira de identificar os pacientes com maior probabilidade de ter SAHOS antes de encaminhá-los para a PSG. Estudos anteriores já utilizaram, para este fim, métodos como oximetria de pulso noturna, curvas de fluxo-volume e ressonância magnética das vias aéreas superiores para avaliar a distribuição de gordura nessa região (18-20). O Clinical Practice Review Committee of the American Academy of Sleep Medicine, em 2003, sugeriu uma série de sinais e sintomas que, usados em combinação, poderiam auxiliar na determinação de quais pacientes deveriam ser investigados para SAHOS (21). No entanto, nem todos os pacientes com SAHOS vão ser detectados por esses questionários, e a confiança nesses instrumentos ainda não foi totalmente estabelecida (22).

Alguns estudos vêm tentando avaliar a prevalência e os fatores preditores de SAHOS na subpopulação de pacientes obesos graves a serem submetidos à cirurgia bariátrica. Frey e Pilcher (23) mostraram uma prevalência de SAHOS de $71 \%$ em pacientes avaliados para este tipo de cirurgia, e chamaram a atenção para a necessidade de um baixo índice de suspeição para solicitação de PSG durante o período de avaliação pré-operatória para este tipo de cirurgia. Nesse estudo, não foi demonstrada relação entre a severidade da doença e o IMC, a Escala de Epworth ou a classificação de Mallampati para as vias aéreas. O'Keeffe e Patterson (9) encontraram uma prevalência de 
SAHOS de $77 \%$ na sua coorte, sendo que apenas $15,3 \%$ tiveram esse diagnóstico antes da consulta cirúrgica. Também não houve relação entre a presença de SAHOS e o IMC, e os autores sugeriram que a PSG deve ser feita de rotina antes da cirurgia bariátrica. Por último, Serafini e cols. (24) também não encontraram relação entre a Escala de Epworth, a presença de ronco e o IMC com o IAH, indicando a necessidade de sempre realizar uma PSG em pacientes obesos graves antes da cirurgia para graduar a severidade da doença.

A fraca correlação entre a presença de sintomas e o IAH já foi relatada em estudos prévios $(25,26)$. Dixon e cols. (27), em um estudo envolvendo uma população selecionada de obesos com sintomas de distúrbios do sono, encontraram que o único sintoma que teve valor preditivo para o IAH foi a presença de apnéia observada durante o sono. Nesse estudo, foram encontrados, ainda, outros fatores que se relacionaram independentemente com a severidade da SAHOS, como circunferência do pescoço, sexo masculino, idade, IMC, níveis séricos de insulina e hemoglobina glicosilada. Os autores sugeriram uma correlação entre a presença de síndrome metabólica e SAHOS e formularam um escore para avaliar o risco de SAHOS e limitar o número de pacientes encaminhados para PSG. Esses resultados foram, no entanto, baseados em uma população selecionada de obesos e devem ser interpretados com cautela antes de serem generalizados para outras populações.

Os resultados encontrados neste estudo mostram que devemos ter um maior cuidado na avaliação pré-operatória dos obesos mórbidos, dada a importância e os benefícios do diagnóstico e tratamento precoce da SAHOS no pré e pós-operatório imediato. Este estudo contribui para a discussão, à medida que apresenta um percentual significativo de pacientes obesos com indicação de cirurgia bariátrica, que são assintomáticos, mas que apresentaram alterações nos índices respiratórios polissonográficos.

\section{REFERÊNCIAS}

1. Gibson GJ. Obstructive sleep apnea syndrome: underestimated and undertreated. Br Med Bull 2005;72:49-65.

2. Young T, Palta M, Dempsey J, Skatrud J, Weber S, Badr S. The occurrence of sleep-disordered breathing among middleaged adults. N Engl J Med 1993;328:1230-5.

3. Bresnitz E. Epidemiology of obstructive sleep apnea. Epidemiol Rev 1994; 16:210-27.

4. Strohl KP, Redline S. Recognition of obstructive sleep apnea. Am J Respir Crit Care Med 1996;154:279-86.

5. Willett WC, Dietz WH, Colditz GA. Guidelines for healthy weight. N Engl J Med 1999;341:427-34.

6. Daltro $\mathrm{CHC}$, Fontes FHO, Santos-Jesus R, Grgório PB, Araújo LMB. Síndrome da apnéia e hipopnéia obstrutiva do sono: associação com obesidade, gênero e idade. Arq Bras Endocrinol Metab 2006;50:74-81.
7. Gami AS, Caples SM, Somers VK. Obesity and obstructive sleep apnea. Endocrinol Metab Clin North Am 2003;32:869-94.

8. Benumof JL. Obstructive sleep apnea in the adult obese patient: implications for airway management. J Clin Anesth 2001;13:144-56.

9. Yaggi HK, Concato J, Kernan WN, Lichtman JH, Brass LM, Mohsenin V. Obstructive sleep apnea as a risk factor for stroke and death. N Engl J Med 2005;353:2034-41.

10. O'Keeffe T, Patterson EJ. Evidence supporting routine polysomnography before bariatric surgery. Obes Surg 2004; $14: 23-6$

11. World Health Organization. Obesity: preventing and managing the Global Epidemic. Report of a WHO Consultation on obesity. Geneva: WHO; 1997.

12. Johns MW. A new method for measuring daytime sleepiness: the Epworth sleepiness scale. Sleep 1991;14:540-5.

13. Sociedade Brasileira de Sono. Consenso brasileiro em ronco e apnéia do sono. São Paulo: SBS, 2000.

14. Rechtschaffen $A$, Kales $A$. A manual of standardized terminology, techniques, and scoring system for sleep stages in human subjects. Los Angeles: Brain Information Service, UCLA, 1968.

15. American Academy of Sleep Medicine Task Force. Sleeprelated breathing disorders in adults: recommendations for syndrome definition and measurement techniques in clinical research. Sleep 1999;22:667-89.

16. Deutzer J. Potential complications of obstructive sleep apnea in patients undergoing gastric bypass surgery. Crit Care Nurs 2005;28:293-9.

17. Cartagena R. Preoperative evaluation of patients with obesity and obstructive sleep apnea. Anesthesiol Clin North Am 2005;23:463-78.

18. Chesson AL, Ferber RA, Fry JM, Grigg-Damberger M, Hartse KM, Hurwitz TD, et al. Practice parameters for the indications for polysomnography and related procedures. Sleep 1997;20:406-22.

19. Bell RL, Rosenbaum SH. Postoperative considerations for patients with obesity and sleep apnea. Anesthesiol Clin North Am 2005;23:493-500.

20. Rauscher H, Popp W, Zwick H. Model for investigating snorers with suspected sleep apnoea. Thorax 1993;48:275-9.

21. Shelton KE, Woodson $H$, Gay S, Suratt PM. Pharyngeal fat in obstructive sleep apnea. Am Rev Respir Dis 1993;148:462-6.

22. Meoli AL, Rosen CL, Kristo D, Kohrman M, Gooneratne N, Aguillard RN, et al. Upper airway management of the adult patient with obstructive sleep apnea in the perioperative period - avoiding complications. Sleep 2003;26:1060-5.

23. Kump K, Whalen C, Tishler PV, Browner I, Ferrette V, Strohl KP, et al. Assessment of the validity and utility of a sleep-symptom questionnaire. Am J Respir Crit Care Med 1994;150:735-41.

24. Frey WC, Pilcher J. Obstructive sleep-related breathing disorders in patients evaluated for bariatric surgery. Obes Surg 2003;13:676-83.

25. Serafini FM, MacDowell Anderson W, Rosemurgy AS, Strait T, Murr MM. Clinical predictors of sleep apnea in patients undergoing bariatric surgery. Obes Surg 2001;11:28-31.

26. Broussolle C, Piperno D, Gormand F, Cambursano H, Berthier M, Perrin-Fayolle M, et al. Sleep apnea syndrome in obese patients: are there any predictive factors? Rev Med Interne 1994; 15:161-5.

27. Dixon JB, Schachter LM, O'Brien PE. Predicting sleep apnea and excessive day sleepiness in the severely obese - Indicators for polysomnography. Chest 2003;123:1134-41.

Endereço para correspondência:

Almir Galvão Vieira Bitencourt

Rua Vanderlei Pinho 181, ap. 802

41815-270 Salvador, BA

Fax: (71) 3245-0858

E-mail: almirgvb@yahoo.com.br / almirgvb@oi.com.br 